\title{
CDISC SEND Trial Summary Parameter Short Name Terminology
}

National Cancer Institute

\section{Source}

National Cancer Institute. CDISC SEND Trial Summary Parameter Short Name

Terminology. NCI Thesaurus. Code C90009.

The terminology that includes concepts relevant to the Clinical Data Interchange Standards Consortium (CDISC) Standard for the Exchange of Non-clinical Data (SEND) trial summary parameter short names. 